\title{
Broadband lasers to detect and cool the vibration of cold molecules
}

\author{
Matthieu Viteau ${ }^{a}$, Amodsen Chotia $^{a}$, Dimitris Sofikitis ${ }^{a}$, \\ Maria Allegrini ${ }^{a, b}$, Nadia Bouloufa $^{a}$, Olivier Dulieu ${ }^{a}$, \\ Daniel Comparat ${ }^{a}$ and Pierre Pillet $^{a}$ \\ ${ }^{a}$ Laboratoire Aimé Cotton, CNRS, Univ Paris-Sud, \\ Bât. 505, 91405 Orsay, France. \\ E-mail: pierre.pillet@lac.u-psud.fr \\ ${ }^{b}$ CNISM, Physics Department, Pisa University, \\ Largo Pontecorvo, 356127 Pisa, Italy.
}

May 4, 2009

\begin{abstract}
By using broadband lasers, we demonstrate the possibilities to control the cold molecules formed via photoassociation. We present first a detection REMPI scheme [1] to systematically investigate the mechanisms of formation of ultracold $\mathrm{Cs}_{2}$ molecules in deeply bound levels of their electronic ground state $\mathrm{X}^{1} \Sigma_{g}^{+}$. This broadband detection scheme could be generalized to other molecular species. Then we report a vibrational cooling technique trough optical pumping obtained by using a shaped mode locked femtosecond laser [2]. The broadband femtosecond laser excites the molecules electronically, leading to a redistribution of the vibrational population in the ground state via a few absorption - spontaneous emission cycles. By removing the laser frequencies corresponding to the excitation of the $v=0$ level, we realize a dark state for the so-shaped femtosecond laser, yielding with the successive laser pulses to an accumulation of the molecules in the $v=0$ level, i.e. a laser cooling of the vibration. The simulation of the vibrational laser cooling allows us to characterize the criteria to extend the mechanism to other molecular species. We finally discuss the generalization of the technique to the laser cooling for the rotation of the molecule.
\end{abstract}

\section{Introduction}

The control of the dynamics of a quantum system is today a crucial goal in both physics and chemistry [3]. The precise control of both of internal and external degrees of freedom of a molecule should open fascinating new fields of research, which motivate the important activities developed in the cold-molecule domain. To prepare robust samples of trapped ultracold ground-state molecules with neither vibration, nor rotation is expected to lead to significant advances in molecular spectroscopy, molecular clocks, fundamental test in physics as electron-dipole moment, chirality or variation of some fundamental constants, collision, super or controlled photo-chemistry studies, and also 
in quantum computation with the use of polar molecules $[4,5] \ldots$ The difficulties for extending laser cooling techniques from atoms to molecules [6] is linked to the lack of isolated and closed two-level schemes in the molecular systems. The large number of rovibrational levels of a molecules makes non-reasonable the addition of repumper lasers as in the case of atoms to close perfectly such a transition. An interesting alternative could be in the use of a laser with a large spectral band able to excite simultaneously several transitions between rovibrational levels of two different electronic potentials of the molecules.

The interest of the use of such broadband lasers has already been demonstrated for two applications with translationally cold molecules formed via photoassociation of cold cesium atoms. Both applications are linked to the distribution of the populations of the so-prepared molecules in a large number of the different ro-vibrational levels. The first one concerns the REMPI (Resonantly-Enhanced Multi-Photon Ionization) detection of the translationaly cold molecules. In this case the laser is a dye laser pumped by the second harmonic of a pulsed Nd:YAG laser with a repetition rate of $10 \mathrm{~Hz}$, a temporal duration of the pulses of $10 \mathrm{~ns}$, and a spectral bandwidth of $25 \mathrm{~cm}^{-1}$. The second application is the laser cooling of the internal degree of freedom of the vibration of the molecules, to prepare a molecular sample in the ground state with no vibration [2]. The used laser is a shaped mode locked femtosecond laser, with a repetition rate of 80 $\mathrm{MHz}$, a pulse duration of $100 \mathrm{fs}$, and a $\sigma$-Gaussian bandwidth of $54 \mathrm{~cm}^{-1}$.

The article is organize as follows. We recall first the dynamics of photoassociation of cold atoms leading to the formation of translationaly cold molecules in several ro-vibrational levels of the ground state or of the lowest triplet state. In the next paragraph, we expose an efficient broadband REMPI process well-adapted to the detection of such cold molecules. Then we report the use of broadband shaped femtosecond sources for the laser cooling of the vibration of these translationally cold molecules. We analyze the criteria for extending the method to any molecular species and the possibilities for realizing the cooling of the rotation of the molecule. In conclusion, we discuss further applications for the manipulation of molecules by broadband lasers.

\section{Cold molecules formed via photoassociation}

The photoassociation has today been demonstrated for a very large number of homo nuclear or heteronuclear systems. Cold ground-state molecules have been observed in many cases. The here reported experiments concern the case of the cesium atom. Our experiment uses Cs but similar experimental results, or theoretical investigation, have been performed in several other systems, including heteronuclear ones. However, we use, in this article, the cesium as an example to describe the photoassociation and refer to more general review, such as $[4,7,8]$, for detail investigation concerning the other systems. The cesium offers many configurations in the formation of cold molecules, corresponding for a pair of atoms to a reaction

$$
C s(6 s, F)+C s(6 s, F)+h \nu_{L} \longrightarrow C s_{2}^{*}\left(\Omega\left(6 s+6 p_{j}\right) ; v, J\right)
$$

Two colliding Cs atoms in an hyperfine level $F$ of their ground state $6 s$ absorb one laser photon at frequency $h \nu_{L}$ red-detuned compared to the atomic resonance frequency $\left(6 s+6 p_{j}, j=1 / 2\right.$ or $\left.3 / 2\right)$ to form a molecule in a well defined rovibrational level $(v, J)$ of an excited molecular state $\Omega$ correlated to one of the asymptotes $\left(6 s+6 p_{1 / 2}\right)$ or 
$\left(6 s+6 p_{3 / 2}\right)$. In cold thermal samples, the resolution of the photoassociation process is limited by the width of the statistical distribution of the relative kinetic energy of the colliding atoms, which is of the order of $k_{B} T\left(k_{B}\right.$ is the Boltzman constant and $T$ the temperature of the atomic sample). Due to the extremely narrow width of the thermal distribution of ultracold atoms $\left(k_{B} T \sim 2 \mathrm{MHz}\right.$ at $\left.T \sim 100 \mu \mathrm{K}\right)$, smaller than any other relevant energy of the system like the molecular level spacing, photoassociation with cold atoms has been proved to be a powerful tool for high-resolution molecular spectroscopy, in particular for the whole class of alkali atoms from Li to Cs $[4,7,8]$. It has given access to the previously unexplored domain of molecular dynamics at distances well beyond those of well-known chemical bonds. Indeed, a pair of identical ground state atoms interact at large interatomic distances $R$ through their Van der Waals interaction behaving as $R^{-6}$; when the atom pair is excited, the dipole-dipole interaction is dominant, and varies as $R^{-3}$. Vibrational levels with a very large elongation (from a few times ten up to a few hundred atomic units) are then efficiently populated by photoassociation, as can be understood from Fig.1 (a) where typical radial wavefunctions for the initial collision state and for the final vibrational level are represented. In a perturbative approach and under the assumption of an $R$-independent dipole transition moment, the photoassociation rate is proportional to the squared overlap integral between radial wavefunctions. Due to the very different oscillatory pattern of the wavefunctions at large distances, we can show that the overlap integral is proportional to $\left|\psi_{\alpha}\left(R_{T}\right)\right|^{2}$ where $\psi_{\alpha}$ is the initial collision state radial wavefunction, and $R_{T}$ is the outer classical turning point of the photoassociated level. In other words, the photoassociation process occurs at large distance, roughly at the classical outer turning point of the final vibrational level. The $R^{-3}$ behavior of the electronically excited potential curve makes quite efficient the photoassociation process.

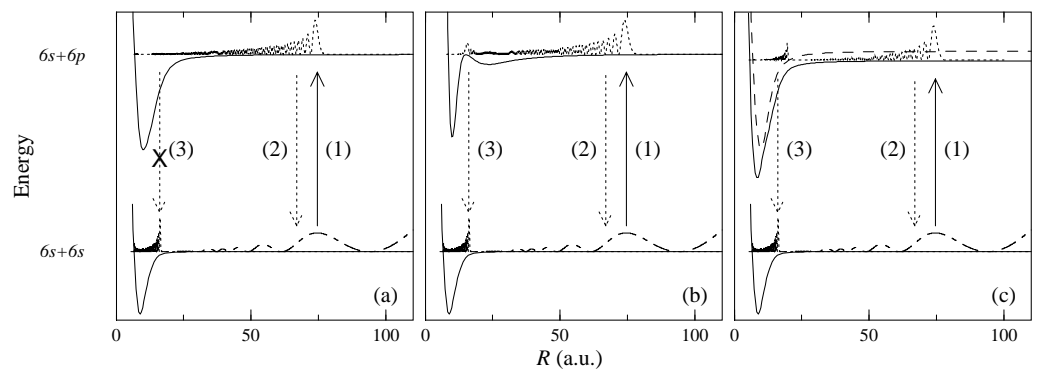

Figure 1: Photoassociation from the cesium $6 s+6 s$ continuum [reaction (1)] to (a) a single excited state; (b) a double-well state [e.g. $\left.0_{g}^{-}, 1_{u}\left(6 s+6 p_{3 / 2}\right)\right] ;$ (c) two coupled states [e.g. $\left.0_{u}^{+}\left(6 s+6 p_{1 / 2,3 / 2}\right)\right]$. The system decays by spontaneous emission either back to the continuum [reaction (2)] or to a bound level of the ground state [reaction (3)]. For case (a), reaction (3) is usually unlikely.

The electronically excited molecules created by photoassociation have a short lifetime of a few tens of nanoseconds due to spontaneous emission. Most often they decay back to a pair of "hot" atoms (i.e. with a large relative kinetic energy). Most experimental photoassociation setups use a magneto-optical trap illuminated by a photoassociation laser. The spontaneous decay provides the detection method of the photoassociation signal: it results in decreasing the intensity of the trap fluorescence due the escape of the hot atoms from the trap. As suggested by Fig.1 (a), deeply bound 
levels of the electronic states correlated to the ground state asymptote cannot be easily populated by spontaneous decay due to the poor overlap of the corresponding radial wave functions, meaning poor Franck-Condon factors. The issue is now to find situations where the probability density of the radial motion can be transferred towards small interatomic distances, to ensure a good spatial overlap with the vibrational wave functions of the deeply bound levels of the lowest potential curves. From a classical point of view, the ideal vibrational motion of the PA molecule should slow down at least in the intermediate distance range (say, around 15-20 atomic units) to let spontaneous decay occur before going back towards the long distance range. This solution has been demonstrated for the first time in 1998, starting from an ensemble of cold cesium atoms [9]. Fig.1 (b) shows a scheme for a double-well configuration corresponding to the $0_{g}^{-}$ and $1_{u}$ double-well potential curves correlated to the $\operatorname{Cs}(6 s)+\operatorname{Cs}\left(6 p_{3 / 2}\right)$ limit. These peculiar states, known as pure long-range molecules, result from the competition of spin-orbit interaction and long-range dipole-dipole interaction. The slow $R$-variation of left edge of the outer potential well induces a "speed bump" in the distance range appropriate for the radiative decay towards stable vibrational levels. Fig.1 (c) illustrates another efficient formation mechanism involving resonances between vibrational levels of different electronic states coupled through a non-Born-Oppenheimer interaction. Such a situation involving internal couplings has been demonstrated for the $0_{u}^{+}$ molecular symmetry in $\mathrm{Cs}_{2}$ but also in $\mathrm{Rb}_{2}$ and KRb [10]. Such a pattern is very common in molecular systems and opens many routes for the formation of cold molecules in photoassociation experiments.

\section{Broadband detection of cold molecules}

The details of the experimental setup for photoassociation of cold atoms in a Cs vaporcell magneto-optical trap can be found in the reference [9]. The photoassociation of cold cesium atoms is achieved with a cw Titanium:Sapphire laser (intensity $300 \mathrm{~W} . \mathrm{cm}^{-2}$ ), pumped by an Argon-ion laser, exciting molecules which can decay by spontaneous emission into vibrational levels of the molecular ground state $X^{1} \Sigma_{g}^{+}$, (hereafter referred to as $\mathrm{X}$ ), or of the lowest triplet state $a^{3} \Sigma_{u}^{+}$. A key point of these experiments is the detection mechanism. Formation of ultracold molecules via photoassociation is demonstrated by using a very sensitive REMPI (Resonant Enhanced MultiPhoton Ionization) detection. Fig.2 (a) shows the potential involved in the REMPI process [2]. The cold molecules are ionized in $\mathrm{Cs}_{2}^{+}$ions by using a pulsed dye laser $(7 \mathrm{~ns}$ duration, $1 \mathrm{~mJ}$ energy, focused to a $1 \mathrm{~mm}^{2}, 15 \mathrm{GHz}$ resolution) pumped by the second harmonic of a Nd-YAG laser, at a $10 \mathrm{~Hz}$ repetition rate. Fig. 3 shows the REMPI spectrum obtained by scanning the frequency of the pulsed REMPI laser in the range $13500-14500$ $\mathrm{cm}^{-1}$, when the molecules are formed via the photoassociation of the vibrational level, $v=79$, of the state $0_{g}^{-}\left(6 s+6 p_{3 / 2}\right)$. For frequencies above $13850 \mathrm{~cm}^{-1}$, the scan consists of many densely located molecular lines. The REMPI process consists in a first resonant step corresponding to transitions between ro-vibrational levels of the lowest triplet state $a^{3} \Sigma_{u}^{+}$and the $2^{3} \Pi_{g}$ state converging to the dissociation limit $6 s+5 d$. The second step corresponds to a one-photon ionization of the intermediate ro-vibrational level of the $2^{3} \Pi_{g}$ state. After the photoionization laser pulse, a pulsed high electric field $(3 \mathrm{kV} / \mathrm{cm}, 0.5 \mu \mathrm{s})$ is applied at the trap position by means of a pair of grids spaced 15 $\mathrm{mm}$ apart to accelerate the ions. The ions expelled from the photoassociation region in a $6 \mathrm{~cm}$ free field zone constituting a time-of-flight mass spectrometer, useful to sep- 
arate the $\mathrm{Cs}_{2}^{+}$ions from spurious $\mathrm{Cs}^{+}$ions. A pair of microchannel plates detects the ions. This detection scheme is quite sensitive, allowing one to observe up to one unique ion. However, the global efficiency of the process is relatively poor, limited by the ion recollection rate $(80 \%)$, the microchannel plate efficiency (35\%) and the REMPI efficiency itself which is unknown. An estimation of $10 \%$ for the REMPI efficiency is obtained by comparing the $\mathrm{Cs}_{2}^{+}$ion signal with the trap loss fluorescence signal, by using calculated branching ratios between bound-bound and bound-free transitions for the photoassociated molecules [11]. This relatively low rate for the REMPI process is linked to its resonant character, leading to the fact that only a few initially populated ro-vibrational levels, for which the two-photon process is resonant, are efficiently ionized. Nevertheless, the envelope of the photoionization spectrum stays roughly the same for any vibrational levels, which makes the considered REMPI process here welladapted to the analysis of the formed molecules, without knowing a priori the details of the molecular structure.
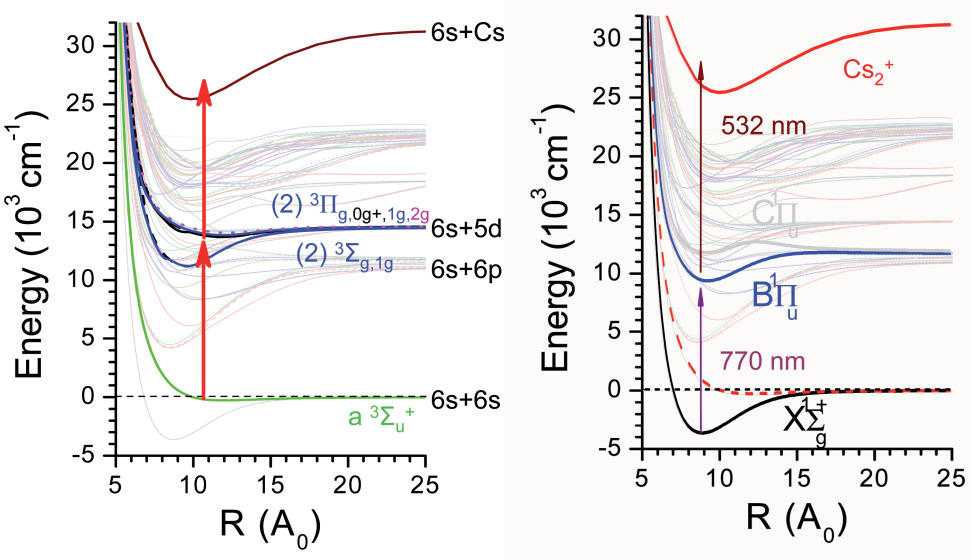

Figure 2: (a) REMPI scheme of the first triplet state with a pulsed dye laser $(\sim 720 \mathrm{~nm}$, (b) REMPI detection scheme of deeply-bound ground state $\mathrm{Cs}_{2}$ molecules with a broadband laser $(770 \mathrm{~nm}$ and $532 \mathrm{~nm})$ via the $B^{1} \Pi_{u}$ state.

For frequencies below $13850 \mathrm{~cm}^{-1}$, the scan consists in a resolved spectrum. The first resonant step of the REMPI process corresponds closely to transitions between ro-vibrational levels of the lowest triplet state $a^{3} \Sigma_{u}^{+}$and the $2^{3} \Sigma_{g}^{+}$state converging to the dissociation limit $6 s+5 d$. The analysis of the lines allows us to conclude that the vibrational level between $v=11$ and 20 of the lowest triplet state, $a^{3} \Sigma_{u}^{+}$, are populated. This part of the spectrum is complementary of the latter one, but if these REMPI resonances give informations of the population in the different vibrational levels, they cannot use for the research of mecanisms in the formation of cold molecules. To conclude at this point of the article, the detected cold molecules are vibrationally excited in the lowest triplet state. Molecules in singlet ground state, as those obtained 
via the photoassociation in the $1_{u}\left(6 s+6 p_{3 / 2}\right)$ and $0_{u}^{+}\left(6 s+6 p_{1 / 2}\right)$ states, are also detected. In both cases, they are in highly excited vibational levels.
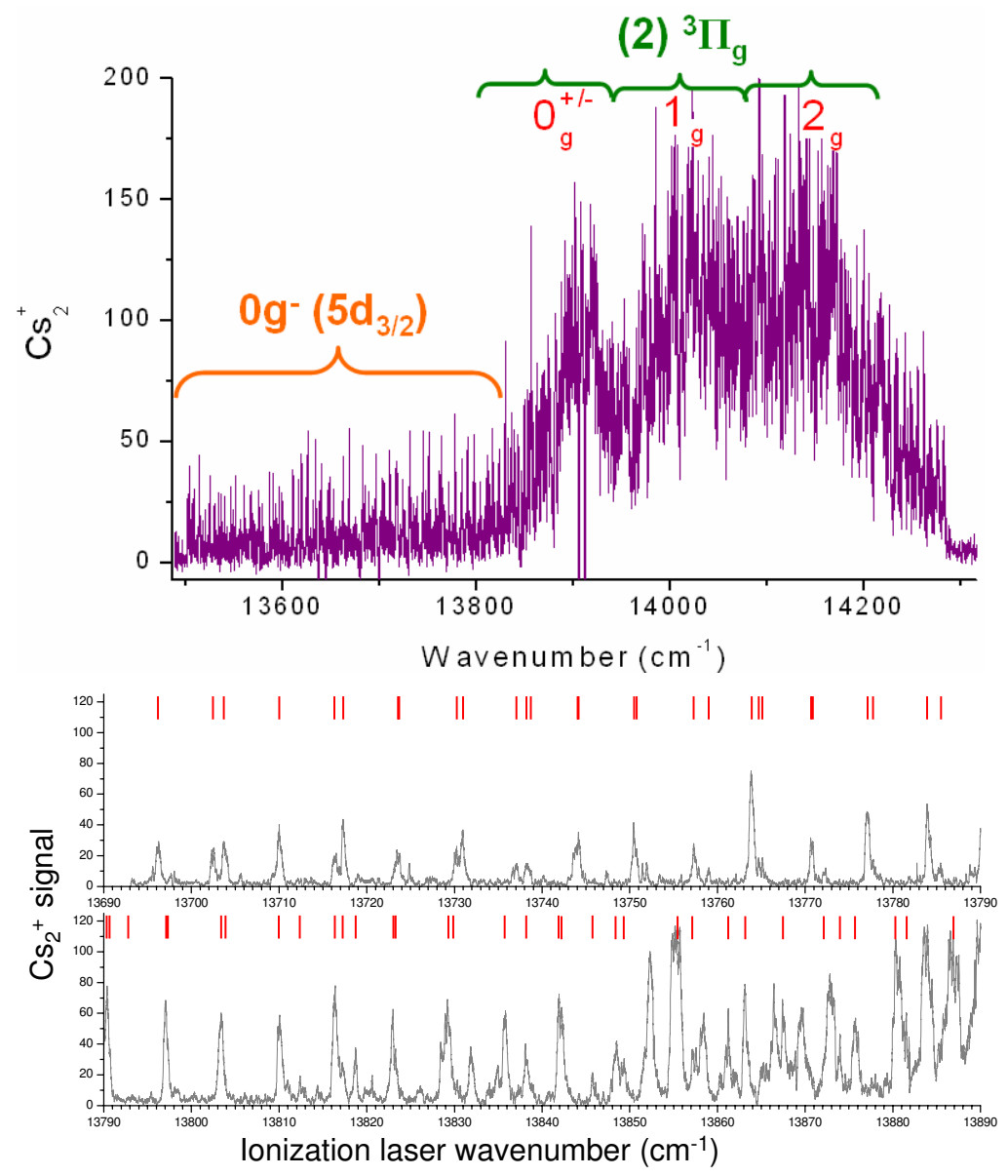

Figure 3: (a) Photoionisation spectrum of $\mathrm{Cs}_{2}$ cold molecule formed after spontaneous emission from the vibrational level, $v=79$, in $0_{g}^{-}\left(6 s+6 p_{3 / 2}\right)$ state. (b) Part of the spectrum where each identified transition are indicated by a line. The ionisation, in this part of the spectrum is obtain via the state $0_{g}^{-}\left(6 s+5 d_{3 / 2}\right)$.

The research of molecule formation schemes based on photoassociation necessitates a general and systematic detection method, without knowing a priori the details of the molecular structure. The proposed method is based on a detection procedure which does not select the population of a particular bound level. In order to observe deeply-bound molecules in the $X$ ground state, which could result from an a priori unknown mechanism, we set up a broadband REMPI detection through vibrational levels $v_{B}$ of the spectroscopically known $B^{1} \Pi_{u}$ excited state [12] (refereed to as the $B$ state). The two-photon transition is induced by a pulsed dye laser (LDS751 dye, wavelength $\sim 770 \mathrm{~nm}$, pulse energy $\sim 1 \mathrm{~mJ}$, focused waist $\sim 500 \mu \mathrm{m})$ and by the pump laser (532 nm wavelength) as illustrated in Fig.2 (b). The major advance of the method compared to previous one is the broadband detection of the formed ultracold molecules. 
We replaced the grating in the pulsed dye laser cavity by a less dispersive prism, which broadens its linewidth from $\sim 0.05 \mathrm{~cm}^{-1}$ to $\sim 25 \mathrm{~cm}^{-1}$. We display in Fig.4 the results of a modeling of the ionization process, for both narrowband (panel a) and broadband (panel b) schemes. We assume that the ionization probability due to second (532 nm) photon is independent of the specific $v_{B}$ level, so that it is proportional to the population of this level induced by the first photon at $770 \mathrm{~nm}$. The excitation probabilities of the $v_{X}$ levels toward the $v_{B}$ levels are obtained from Franck-Condon factors computed for the experimentally known $X$ and $B$ potential curves [13, 12], assuming a constant dipole transition moment. As expected, the narrowband ionization scheme allows the ionization of a single $v_{X}$ level at a given frequency (Fig.2 (a)). In contrast, the broadband scheme involves a laser pulse width of the order of the vibrational spacing of both the $X$ and $B$ states (up to $40 \mathrm{~cm}^{-1}$ ), so that many vibrational $v_{X}$ levels can be ionized in a single shot (Fig.4 (b)). For instance, a laser pulse at $\sim 11730 \mathrm{~cm}^{-1}$ or at $\sim 13000 \mathrm{~cm}^{-1}$ would excite almost all molecules lying in vibrational levels $v_{X}>37$ or $v_{X}<70$ respectively.

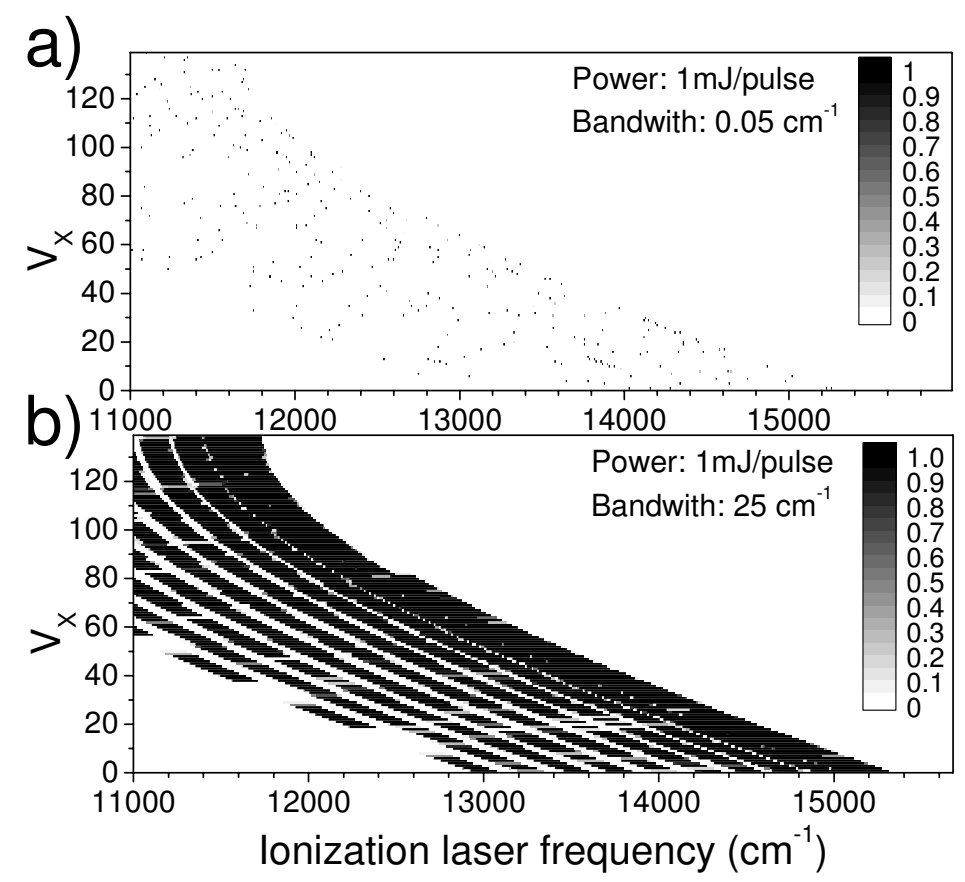

Figure 4: (a) and (b) Comparison of transition probabilities (in level of grey) for the transitions at energy $E_{X-B}$ between vibrational levels of the $X\left(v_{X}\right)$ and $B$ states, for a laser linewidth of $0.05 \mathrm{~cm}^{-1}$ (a) and of $25 \mathrm{~cm}^{-1}(\mathrm{~b})$, with identical power $(1 \mathrm{~mJ} / \mathrm{pulse})$. The probability is put to unity for a saturated transition.

Choosing the latter energy range for the broadband detection laser $\left(\sim 13000 \mathrm{~cm}^{-1}\right)$ and scanning the photoassociation laser frequency over a few wavenumbers below the $6 s+6 p_{3 / 2}$ dissociation limit, we discovered several intense photoassociation lines labeled with crosses in Fig.5, revealing a large number of ultracold molecules formed in low $\left(v_{X}<70\right)$ vibrational levels of the $X$ state. These detected singlet molecules 
were present in our previous experiments performed in the same photoassociation energy range [9], but our previous narrowband REMPI detection scheme (wavelength $\sim 720 \mathrm{~nm}$ ), optimized to detect triplet $\mathrm{a}^{3} \Sigma_{u}^{+}$molecules, was blind for these singlet molecules (see lower spectrum of Fig. 5). A new mechanism for the formation of cold molecules has so been identified. We depict the process as follows: the PA laser excites the atom pair into a bound level of the lowest $1_{g}\left(6 s+6 p_{3 / 2}\right)$ long-range potential curve (Fig.6 (a)), which is coupled at short distances to the lowest $1_{g}\left(6 s+6 d_{5 / 2}\right)$ potential curve, through several avoided crossings induced by spin-orbit interaction (situation similar to that of Fig.1 (c)).

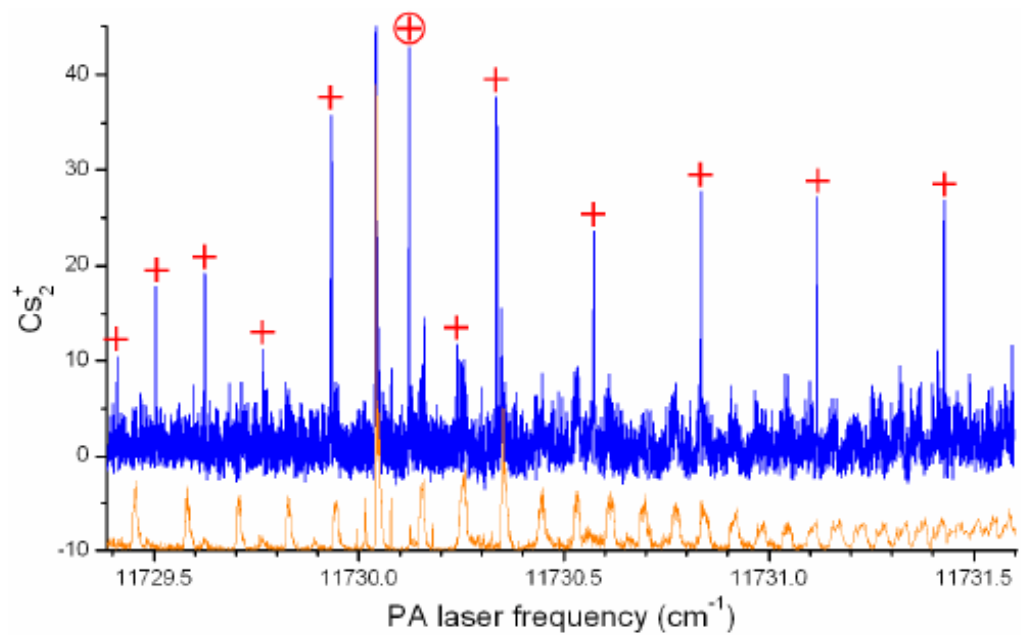

Figure 5: Upper trace: $\mathrm{Cs}_{2}^{+}$ion spectrum recorded after scanning the frequency of the PA laser below the $6 s+6 p_{3 / 2}$ dissociation limit, and using the broadband REMPI detection laser with energy around $13000 \mathrm{~cm}^{-1}$. The crosses label the previously unobserved PA lines. Lower trace: $\mathrm{Cs}_{2}^{+}$ion spectrum obtained using the conventional narrowband REMPI detection [9], displayed with an offset of 10 ions for clarity.

\section{Laser cooling of the vibration of molecules}

To determine precisely the internal state of these formed molecules we performed a more conventional REMPI spectra using a narrowband (DCM) dye laser (wavelength $\sim 627 \mathrm{~nm}$ ). We fixed the PA laser energy on the most intense line of Fig.5 (at $11730.1245 \mathrm{~cm}^{-1}$ ), about $2 \mathrm{~cm}^{-1}$ below the $6 s+6 p_{3 / 2}$ asymptote. Then we scanned the ionization laser frequency to record the ionization spectrum of the ground state molecules excited through the intermediate $\mathrm{C}^{1} \Pi_{u}$ state (see Fig.6 (b)). Taking advantage of the spectroscopic knowledge of the $X-C$ transitions $[13,14]$, the lines, shown in Fig.7, were easily assigned to transitions from ground state vibrational levels restricted to the range $v_{X}=1$ to $v_{X}=9$. Taking into account the efficiency of the detection [9], the ion signal corresponds to a cumulated formation rate for the $v_{X}<10$ molecules close to $10^{6}$ per second. The next challenge was clearly to prepare these molecules in a well-defined vibrational level. 


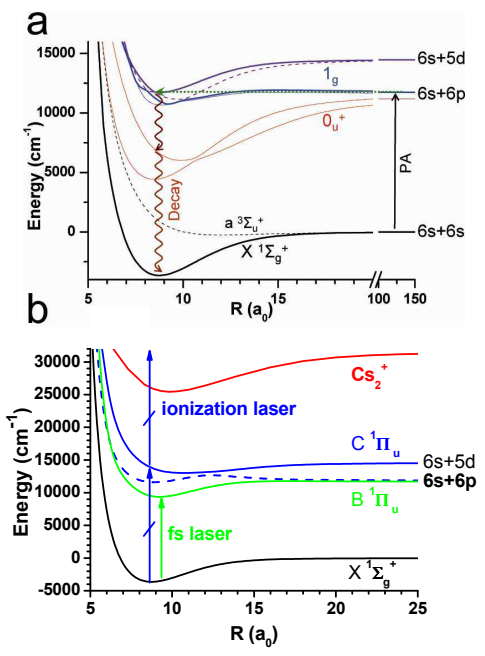

Figure 6: Relevant molecular states of the cesium dimer, converging towards the dissociation limits $6 \mathrm{~s}+6 \mathrm{~s}, 6 \mathrm{~s}+6 \mathrm{p}$, and $6 \mathrm{~s}+5 \mathrm{~d}$. (a) Photoassociation of cold atoms and formation of cold molecules. For the potentials $1_{g}$, long-range radial wavefunction is coupled to short range radial wavefunction by internal coupling of the potentials. The ground-state molecules, $\mathrm{X}^{1} \Sigma_{g}^{+}$, are formed in a cascading spontaneous emission via the $0_{u}^{+}$potentials. (b) RE2PI detection process via the $\mathrm{C}^{1} \Pi_{u}$ state, and transition $\left(\mathrm{X}^{1} \Sigma_{g}^{+}\right.$ towards $\mathrm{B}^{1} \Pi_{u}$ ) of applying the femtosecond laser.

Different schemes have been proposed to favor the formation of cold molecules in their lowest vibrational level. By using a two photon process for photoassociation, a few $v=0$ (no vibration) ultracold ground-state potassium dimers have been observed [15], but several other vibrational levels are populated as well. By transferring a given vibrational level in the lowest vibrational one, cold ground state molecules has been prepared [16]. The Raman photoassociation for preparing ultracold molecules in a well defined level has been studied by different groups. Its efficiency is unfortunately limited, because the so-prepared molecules can be excited again, and spontaneously decay toward other vibrational levels. To go further, an exciting direction is vibrational laser cooling, which would consist in transferring all the populations of the different vibrational levels in the lowest one. Such a goal is not possible without carrying away the entropy from the molecule via dissipative spontaneous-emission process. Before this work, laser cooling of vibrational degrees of freedom of cold molecules has never been experimentally realized. Several theoretical approaches have been proposed to favor the spontaneous emission towards the lowest ro-vibrational level, as for instance the use of an external cavity [17].

The first step in the vibrational laser cooling is in the realization of the optical pumping applied to the molecules by using a femtosecond laser. We have demonstrated the transfer of population from the vibrational levels of cold singlet-ground-state $\mathrm{Cs}_{2}$ molecules prepared via photoassociation, towards the level, $v=0$, with no vibration. The main idea is the use a broadband laser tuned to the transitions $\mathrm{X}^{1} \Sigma_{g}^{+}\left(v_{X}\right)$ towards 


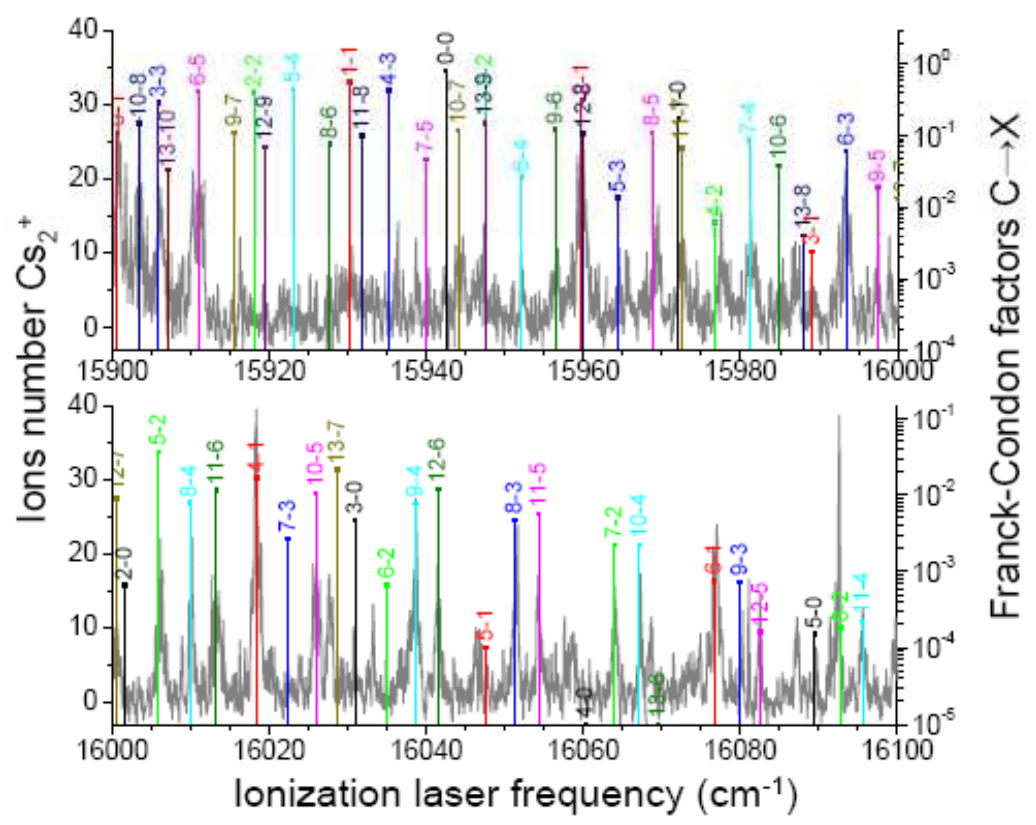

Figure 7: $\mathrm{Cs}_{2}^{+}$ion count (left vertical axis) resulting from a narrowband REMPI detection (frequency $\sim 627 \mathrm{~nm}$ ). The PA laser energy is fixed at $11730.1245 \mathrm{~cm}^{-1}$ corresponding to the position of the most intense line marked with a circled cross in Fig. 5. Transition labels $v_{C}-v_{X}$ are extracted from computed Franck-Condon Factors (right vertical axis) between vibrational levels of the spectroscopically known $C$ and $X$ states.

$\mathrm{B}^{1} \Pi_{u}\left(v_{B}\right)$ between the different vibrational levels of the ground state and of the electronically excited one. With the successive laser pulses, the absorption - spontaneous emission cycles lead through optical pumping to a redistribution of the vibrational population in the ground state. By removing the laser frequencies corresponding to the excitation of the $v_{X}=0$ level, we realize a dark state for the shaped femtosecond laser, leading to an accumulation of the molecules in the $v_{X}=0$ level, meaning vibrational laser cooling. A similar concept of dark state is introduced in theoretical articles $[18,19]$, where the vibration of the molecule is manipulated through quantum interferences between the different transitions. Interplay of control laser fields and spontaneous emission has been investigated for rotational or vibrational cooling. The mechanism proposed is called, molecular vibration selective coherent population trapping (MVSCPT), in analogy to the corresponding mechanism of velocity selective coherent population trapping (VSCPT) in atoms for sub-Doppler cooling of translations [19].

The used broadband laser is a Tsunami mode locked femtosecond laser from Spectra Physics. The repetition rate is $80 \mathrm{MHz}$, the average power $2 \mathrm{~W}$, the pulse duration $100 \mathrm{fs}$, and the $\sigma$-gaussian bandwidth $54 \mathrm{~cm}^{-1}$. The femtosecond laser is tuned (wavelength $767 \mathrm{~nm}$ ) to the electronic transitions $\mathrm{X}^{1} \Sigma_{g}^{+}\left(v_{X}\right)$ towards $\mathrm{B}^{1} \Pi_{u}\left(v_{B}\right)$ of the cesium dimer (see Fig.6b). The low vibrational levels $\left(v_{X}<10\right)$ can be excited to vibrational levels, $v_{B}$, of the state inducing through optical pumping a redistribution of the pop- 
ulations in the different vibrational levels of the ground state. Without shaping the femtosecond laser, we can observe, depending on the wavelength, a modification of the molecular resonance lines interpreted as a transfer of population between vibrational levels through optical pumping. To control the optical pumping of the molecules, we have shaped the femtosecond laser by suppressing the frequencies corresponding to the excitation of the vibrational level, $v_{X}=0$.

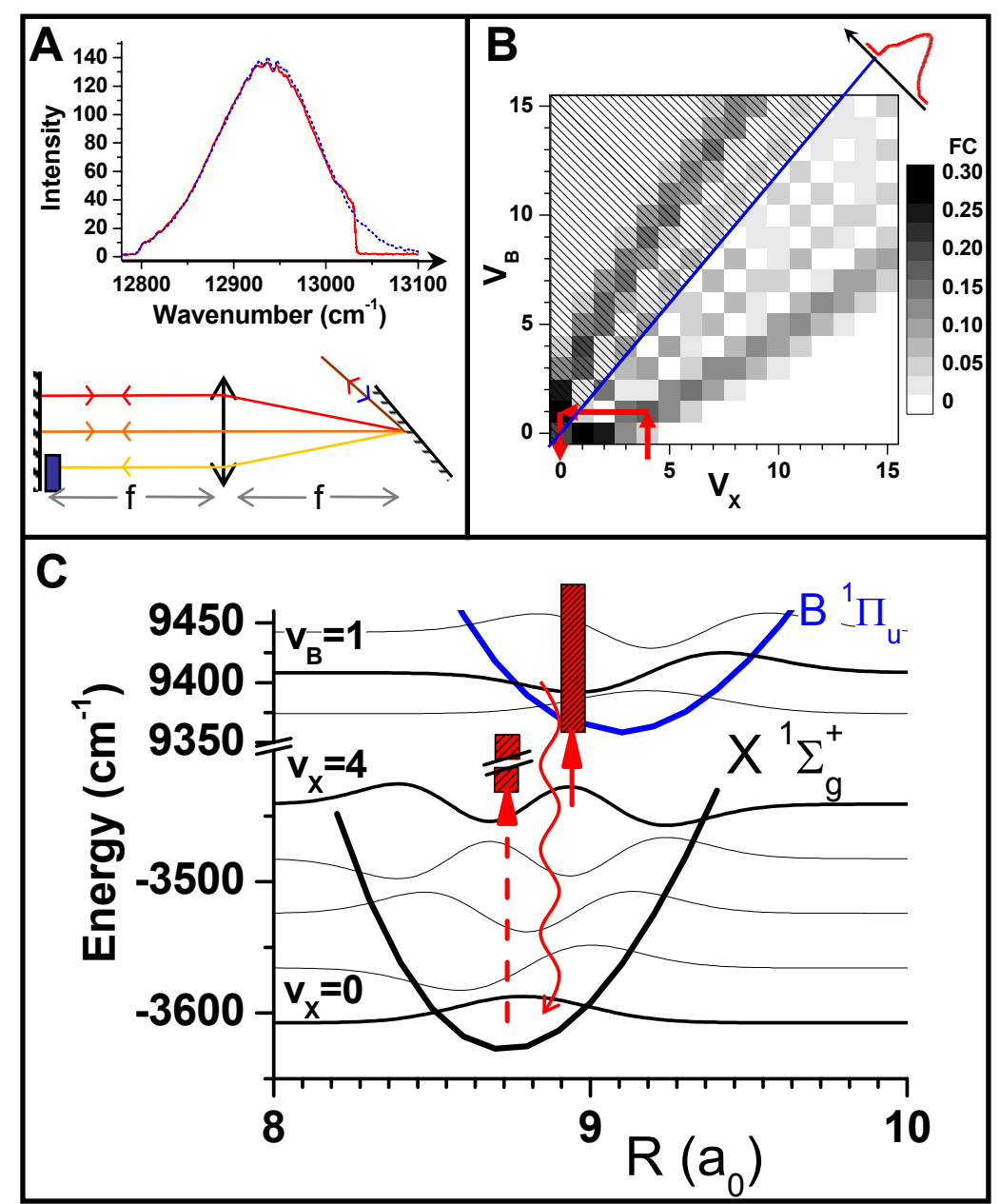

Figure 8: (a) Shaping of the femtosecond laser (see text). (b) Franck-Condon parabola indicating the importance of the Franck-Condon factors (level of grey). (c) Scheme of the optical pumping.

Fig.8 (b) shows the Condon parabola of the Franck-Condon factors for the transitions between the vibrational levels of the states $\mathrm{X}$ and $\mathrm{B}$. The importance of the Franck Condon factors is indicated by the level of grey. Fig.8 (c) shows the overlap between the radial wavefunctions, showing how the Franck Condon factors depend as 
the square of the overlap, on the relative positions of the wells $\mathrm{X}$ and $\mathrm{B}$. The transitions from the $v_{X}=0$ level towards $v_{B}$ levels are at frequencies superior to $13030 \mathrm{~cm}^{-1}$. The spectral shape of the laser does not contain any transitions from $v_{X}=0$ (see Fig.8 (a)). We have used a home made shaper using a grating (1800 lines per $\mathrm{mm}$ ) for diffracting the laser beam and for screening a part of the frequencies. By this way, the vibrational level, $v_{X}=0$, becomes a dark state for the so-shaped laser. If we consider for instance $v_{X}=4$, it is essentially excited into $v_{B}=1$, which decays with a rate of about $30 \%$ in the dark level, $v_{X}=0$, and with a rate of $70 \%$ essentially into the levels $v_{X}=3,4$ or 5 . More generally we understand easily that after a few cycles of absorption of laser light then spontaneous emission that a large fraction of the molecules can be accumulated in the lowest vibrational level, $v_{X}=0$.

Fig.9 shows the experimental results. Adding the shaped laser modifies completely the resolved REMPI spectrum. The resonance lines corresponding to the transition $v_{X}=0 \longrightarrow v_{C}=0-3$, mostly absent in the original spectrum are now very strong. Their broadening correspond to the saturation of the resonance in the REMPI process. The intensity of the lines indicates a very efficient transfer of the molecules into the lowest vibrational level, meaning a vibrational laser-cooling of the molecules. Taking into account the efficiency of the detection $(<10 \%)$, the detected ion signal corresponds to about one thousand molecules in the $v_{X}=0$ level, corresponding to a flux of $v_{X}=0$ molecules of a few $10^{5}$ per second. Fig.10 (a) shows the experimental time evolution of the population in the different vibrational levels. We show that the transfer of population into the $v_{X}=0$ level is almost saturated after the application of 10000 pulses, which requires one hundred microseconds.

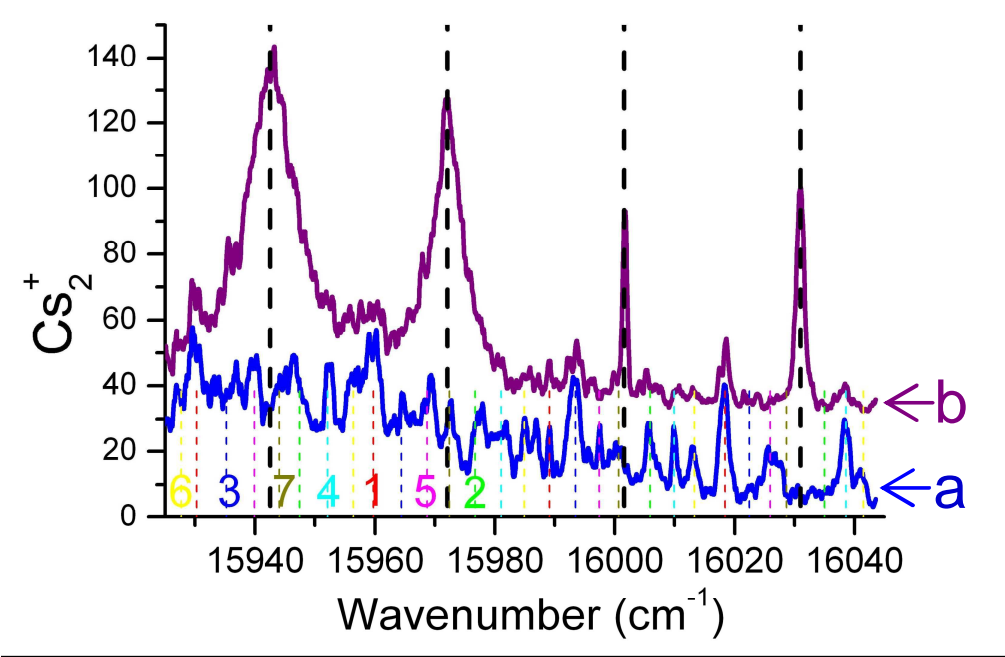

Figure 9: $\mathrm{Cs}_{2}^{+}$ion spectra: (a) without the shaped laser, (b) with the shaped laser. The vibrational levels, $v_{X}$, of the ground state are labeled by different colors. The dashed lines indicate the resonance lines for vibrational transitions $\left(v_{X}\right.$ towards $\left.v_{C}\right)$ between the ground state, $\mathrm{X}^{1} \Sigma_{g}^{+}$, and the electronically excited one, $\mathrm{C}^{1} \Pi_{u}$. The color of the dashed line correspond to this of the involved $v_{X}$. The observed transitions correspond to $\Delta v=\left|v_{C}-v_{X}\right|=0,1,2$ and 3 . 


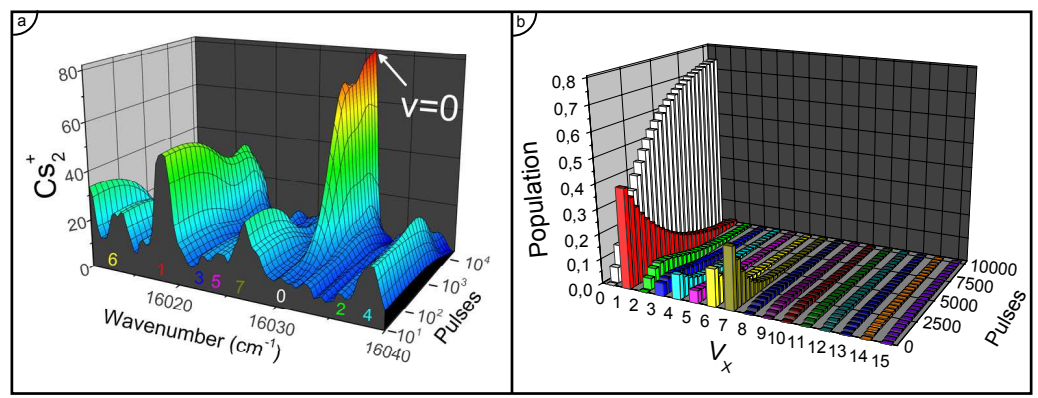

Figure 10: Temporal evolution of the population transfer (a) Compilation of experimental spectrum for different duration of the femtosecond laser (number of pulses). (b) Simulation of the vibrational laser cooling.

\section{Simulation of vibrational laser cooling}

We have modeled the optical pumping in a very simple way. Using the known $\mathrm{X}^{1} \Sigma_{g}^{+}$ and $\mathrm{B}^{1} \Pi_{u}$ potential curves and their rotational constants $[13,12]$, we have calculated the ro-vibrational energy levels. In the perturbative regime, we assume that the excitation probability is simply proportional to the laser spectral density at the transition frequencies, to the Franck-Condon factor [12], and to the Hönl-London factor [?]. We assume a laser spectrum shape very close to the experimental one: average intensity of $150 \mathrm{~mW} / \mathrm{cm}^{2}$ gaussian shape center at $12940 \mathrm{~cm}^{-1}$ with a gaussian linewidth $\sigma=54$ $\mathrm{cm}^{-1}$ and we removed all spectral components, due to the shaping, above $13030 \mathrm{~cm}^{-1}$. After being excited by a pulse and before the shot of the next pulse, we assume a total decay of the excited state population with branching ratio given by the Franck-Condon factors, and the Hönl-London factors. The perturbative regime and the lifetime of the electronically state $\sim 15 \mathrm{~ns}$, close to the period of the pulses $12.5 \mathrm{~ns}$, make reasonable the hypothesis of neglecting any accumulation of coherence due to the excitation by a train of ultrashort pulses [20]. Applying the simple theoretical model shows that the molecules make a random walk, mostly in low vibrational levels, until reaching the $v_{X}=0$ vibrational level. The accumulation of many molecules in the lowest vibrational level occurs with near unit transfer efficiency. Fig.10 (b) shows a simulation of the transfer of the $70 \%$ population into the $v_{X}=0$ level, after 10000 pulses when the molecules are initially in a distribution of vibrational levels simulating the experimental one. The theoretical model gives a good agreement with the data. It indicates that only about 5 cycles absorption - spontaneous emission (the number of necessary laser pulses depends on its intensity) are necessary for a molecules to be transferred in the $v_{X}=0$ level, if the molecules are initially in $v_{X}<10$ vibrational levels. The mechanism could be called, incoherent molecular vibration selective population trapping (incoherent MVSPT), in analogy to the proposed mechanism of the reference [19]. The limitation of the mechanism is in the optical pumping towards high vibrational levels. A broader bandwidth laser could probably increase the population in $v_{X}=0$. Nevertheless the Franck Condon factors favor the accumulation of population in low vibrational levels. 


\section{Simulation for rotational laser cooling}

Can the laser cooling of the vibration to be extended to the rotation of the molecules? For that we need to consider the transitions between the different rotational levels, restricted to $\Delta J=J_{B}-J_{X}=0, \pm 1$. A possibility for laser cooling of the rotation of the molecule, is to shape the laser by removing the frequency corresponding to the transitions $\Delta J=J_{B}-J_{X}=0,+1$, leading with the cycles absorption- spontaneous emission to a decreasing of the average of the principal quantum number $J_{X}$ : i.e. a laser cooling of the rotation.

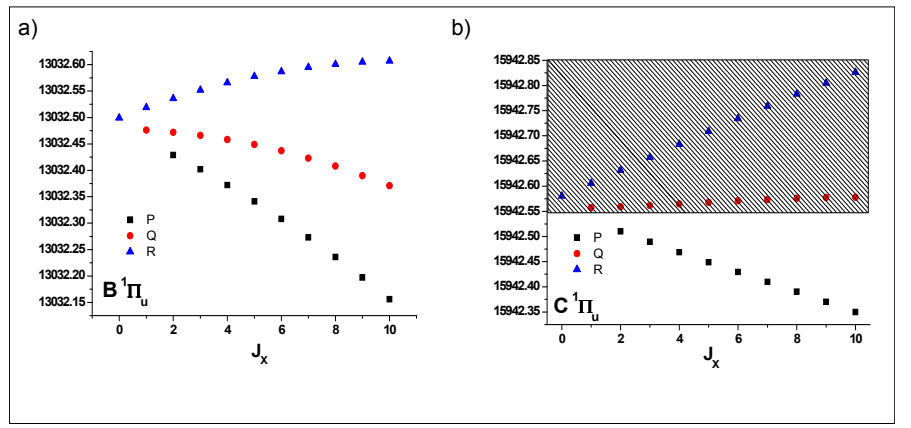

Figure 11: Energy of the different ro-vibrationnel transition $(\Delta J=0 \pm 1$ for the $\mathrm{P}$, $\mathrm{Q}$ and $\mathrm{R}$ branches) (a) with the $B$ state, (b) with the $C$ state. The hatched areas represent a shaping of the laser, to realize a rotational cooling.

Fig.11 shows the energies corresponding to the transitions between the vibrational levels $v_{X}=0$ and $v_{B}=0$. By a blue shaping of the laser, it is not possible to suppress the transitions $\Delta J=J_{B}-J_{X}=0$. The situation is different, if we consider no longer the state, $B^{1} \Pi_{u}$, but the state, $C^{1} \Pi_{u}$. Fig.11 shows that the the transitions $\Delta J=J_{C}-J_{X}=0$ can be easily suppressed. Fig12 shows the results of a simulation where the molecules are first vibrationally cooled by considering the excitation of the state, $B^{1} \Pi_{u}$, then rotationally cooled by considering the excitation of the state $C^{1} \Pi_{u}$.

\section{Conclusion}

We pointed out the interest of the use of broadband sources for cold molecules formed via photoassociation. We demonstrated the ability of a new broadband ionization procedure to detect most of the bound ground state molecules formed in a cold gas. This approach, combined with PA in excited electronic states, provides a general method for 


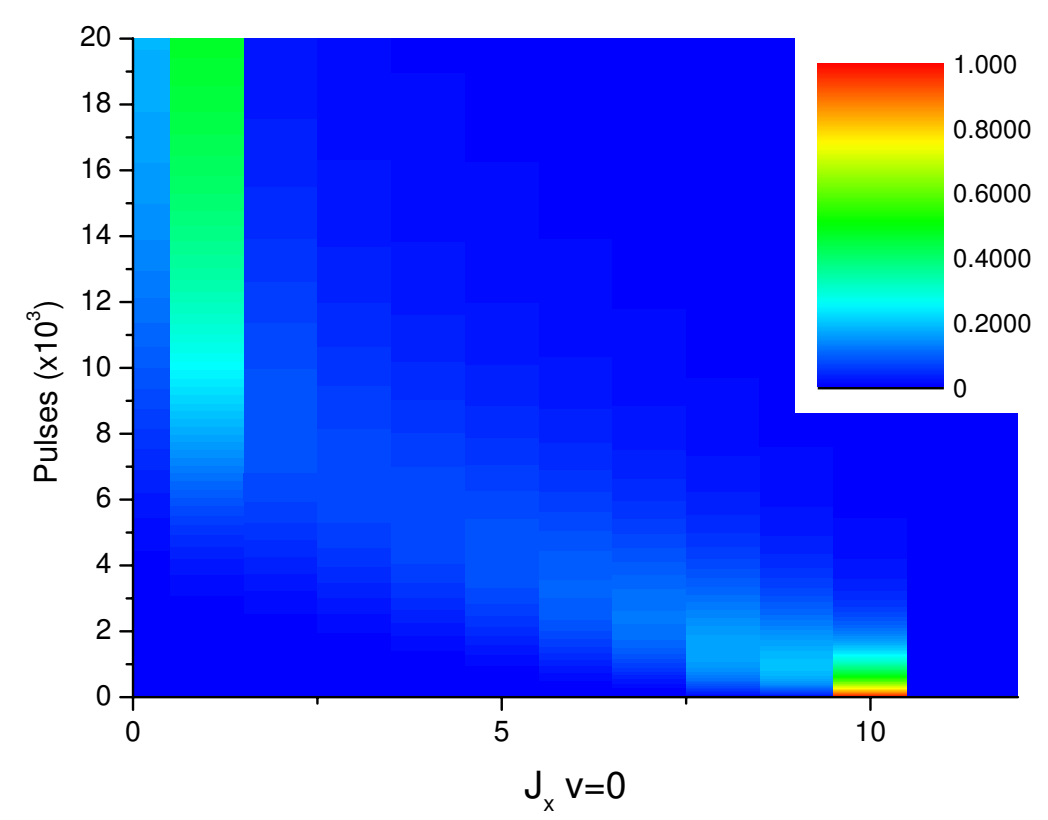

Figure 12: Simulation of the temporal evolution for a ro-vibrational cooling. Rotational cooling, transfer of the populations obtain after the vibrational cooling to the two last rotational level $v_{X}=0-J=0,1$, via the state $C$.

the search of novel paths for formation of cold molecules. Applied to a Cs MOT, this novel approach allowed us to detect deeply bound $\mathrm{Cs}_{2}$ molecules in the $\mathrm{X}^{1} \Sigma_{g}^{+}$state. We show the possibility of the optical pumping of molecules, to modify the distribution of the populations in the vibrational levels. By making the $v=0$ level a dark state, we enable the accumulation of the population in this level, meaning the demonstration of laser cooling for the molecular vibration. The broadband character of the femtosecond laser is however the essential property of our experiment. The possibility to easily shape such a laser is also an important argument for using it, but a continuous broadband laser such as fiber laser or diode laser could may be offer the same potentiality and should be tested in the near future.

In our experiment, we do not presently have the capability of analyzing the rotational populations of the molecules in the vibrational level, $v_{X}=0$, although a specific high resolution detection can be developed. Rotational cooling can be performed in a similar way provided that the laser bandwidth and the experimental ability to shape the laser match the rotational energy spread. The implication of the optical pumping of molecules has important other implications [6]. The possibility of controlling the vibration and the rotation can be used to cool the internal degrees of freedom of polar molecules loaded in an electrostatic trap, after velocity filtering of an effusive molecular beam $[21,22]$.

The trapping of molecules can provide the way to prepare a sample of one million or more molecules. Similar results could also be reached for hetero-nuclear systems and the formation of polar molecules, opening exciting perspectives in quantum information.

This work is supported by the "Institut Francilien de Recherche sur les Atomes 
Froids" (IFRAF). M.A. thanks the EC-Network EMALI. 


\section{References}

[1] M. Viteau, A. Chotia, M. Allegrini, N. Bouloufa, O. Dulieu, D. Comparat, and P. Pillet, Phys. Rev. A, 2009, 79, 021402(R).

[2] M. Viteau, A. Chotia, M. Allegrini, N. Bouloufa, O. Dulieu, D. Comparat, and P. Pillet, Science, 2008, 321, 232-234.

[3] H. Rabitz, R. de Vivie-Riedle, M. Motzkus, and K. Kompa, Science, 2000, 288, $824-828$.

[4] J. Doyle, B. Friedrich, R. V. Krems, and F. Masnou-Seeuws, Eur. Phys. J. D, 2004, 31, 149-164.

[5] R. V. Krems, Int. Rev. Phys. Chem., 2005, 24, $99-118$.

[6] J. T. Bahns, W. C. Stwalley, and P. L. Gould, J. Chem. Phys., 1996, 104, 96899697.

[7] O. Dulieu, M. Raoult, and E. Tiemann, J. Phys. B, 2006, 39(19).

[8] K. M. Jones, E. Tiesinga, P. D. Lett, and P. S. Julienne, Reviews of Modern Physics, 2006, 78, 483-535.

[9] A. Fioretti, D. Comparat, A. Crubellier, O. Dulieu, F. Masnou-Seeuws, and P. Pillet, Phys. Rev. Lett., 1998, 80, 4402-4405.

[10] C. M. Dion, C. Drag, O. Dulieu, B. Laburthe Tolra, F. Masnou-Seeuws, and P. Pillet, Phys. Rev. Lett., 2001, 86, 2253-2256.

[11] C. Drag, B. L. Tolra, O. Dulieu, D. Comparat, M. Vatasescu, S. Boussen, S. Guibal, A. Crubellier, and P. Pillet, IEEE Journal of Quantum Electronics, 2000, 36, 1378-1388.

[12] U. Diemer, R. Duchowicz, M. Ertel, E. Mehdizadeh, and W. Demtröder, Chem. Phys. Lett., 1989, 164, 419-426.

[13] W. Weickenmeier, U. Diemer, M. Wahl, M. Raab, W. Demtröder, and W. Müller, J. Chem. Phys., 1985, 82, 5354-5363.

[14] M. Raab, G. Höning, W. Demtröder, and C. R. Vidal, J. Chem. Phys., 1982, 76, $4370-4386$.

[15] A. N. Nikolov, E. E. Eyler, X. T. Wang, J. Li, H. Wang, W. C. Stwalley, and P. L. Gould, Phys. Rev. Lett., 1999, 82, 703-706.

[16] J. M. Sage, S. Sainis, T. Bergeman, and D. Demille, Phys. Rev. Lett., 2005, 94(20), $203001-+$.

[17] G. Morigi, P. W. H. Pinkse, M. Kowalewski, and R. de Vivie-Riedle, Phys. Rev. Lett., 2007, 99(7), 073001-+.

[18] S. G. Schirmer, Phys. Rev. A, 2000, 63(1), 013407-+.

[19] A. Bartana, R. Kosloff, and D. J. Tannor, Chemical Physics, 2001, 267, 195-207. 
[20] D. Felinto, C. A. C. Bosco, L. H. Acioli, and S. S. Vianna, Optics Communications, 2003, 215, 69-73.

[21] S. A. Rangwala, T. Junglen, T. Rieger, P. W. Pinkse, and G. Rempe, Phys. Rev. A, 2003, 67(4), 043406-- .

[22] T. Rieger, T. Junglen, S. A. Rangwala, P. W. Pinkse, and G. Rempe, Phys. Rev. Lett., 2005, 95(17), 173002-+. 